\title{
A Novel Artifact Reconstruction Method Applied to Blood Pressure Signals *
}

\author{
Javier Rodriguez and Beatriz F. Giraldo, Senior Member, IEEE
}

\begin{abstract}
Physiological records are one of the most relevant elements to obtain objective information from the patients. The presence of artifacts in biomedical signals can give misleading in the analysis of information that these signals give. The blood pressure signal is one of the records clearly affected by different artifacts, especially the ones due from the calibration episodes. We propose a method to reconstruct different episodes of artifacts in these signals. This method is sustained on the detection of the events of the signal, differentiating between to the physiological cycles and the artifacts. The performance of the method is based on the detection of the cycles and artifact's position, the identification of the number of cycles to reconstruct, and the prediction of the cycle model used to generate the missing cycles. The parameter $\Theta_{E}$ represents the difference between the area under the curve when two events are compared. The value of this parameter is low when two similar events are compared like the physiological cycles, whereas it is high comparing a cycle with an artifact. An adaptive threshold is defined to identify the artifact episodes. The number of cycles to reconstruct is generated considering the same number of their neighbours physiological cycles, to left and right, of the original signal. Finally, the performance of the method has been analyzed comparing the number of events and artifacts detected and their correct reconstruction. According to the results, the reconstruction error was less than $1 \%$ in all cases.
\end{abstract}

\section{INTRODUCTION}

A quasi-periodic signal exhibits an irregular periodic behavior, with recurring patterns. Most biomedical signals present this type of behavior given the nature of their physiological processes.

Medicine and technology are becoming more dependent one each other and necessary to be optimally efficient. Analysis of physiological signals is a powerful tool in both clinical diagnosis and health research. The record of biomedical signals allows to obtain patient information.

* This work was supported in part by the Secretariat of Universities and Research of the Department of Economy and Knowledge of the Government of Catalonia (Consolidated research group GRC 2014 SGR 1569), by CERCA Programme / Generalitat de Catalunya, by the Spanish Ministry of Economy and Competitiveness through project DPI201568820-R (MINECO/FEDER).

J. Rodriguez is with Institute for Bioengineering of Catalonia (IBEC), Automatic Control Dept. (ESAII), EEBE, Universitat Politècnica de Catalunya (UPC).

B.F. Giraldo is with Automatic Control Dept. (ESAII), EEBE, Universitat Politècnica de Catalunya (UPC), CIBER de Bioengenieria, Biomateriales y Nanomedicina (CIBER-BBN) and Institute for Bioengineering of Catalonia (IBEC), The Barcelona Institute of Science and Technology, Av. Eduard Maristany, 16. 08019, Barcelona, Spain. (beatriz.giraldo@upc.edu).
However, these signals can be corrupted by noise, artifacts, and missing data. Their presence hinders the application of processing techniques, and their analysis and interpretation.

Some authors have reported this problem in several types of signals and applications. Reconstruction of artifacts in electrocardiogram and blood pressure (BP) signals have been attempted defining a specific model and using its information in correlated signals to perform the reconstruction, or using template matching algorithms $[1,2]$. Other works are based on the use of the stationary subspace analysis [3] and independent component analysis [4 -7] to remove ocular artifacts in electroencephalogram recordings.

Time varying spectral analysis has been applied to reconstruct motion artifacts in the heart rate signal [8]. Empirical mode decomposition has also been used to reconstruct photoplethysmographic signals with artifacts [9]. Most of these methods are valid only to specific type of artifacts. Consequently, the development of efficient algorithms capable of removing or at least identifying artifacts in the recorded data are critically important.

The blood pressure signal recorded using finger cuff is one of the most commonly affected by calibration episodes. Sometimes the correct detection of the pulse in this measurement is not straightforward. Periods of constant cuff pressure are used to adjust the correct unloaded diameter of the finger artery, and the measurement of blood pressure is temporarily interrupted. To study the dynamics of this signal over time, it is necessary to reconstruct the cycles omitted by the calibration. This process is also necessary when the signal shows external artifacts to the registered physiological process. The reconstruction will be possible if the form of the artifact is clearly different from the physiological event.

We present a novel method for reconstruction of the missing cycles of the blood pressure signal, taking their neighbours into account, and preserving the dynamics of the signal according to its physiological behaviour. The performance of the method is based on detecting the cycles and artifact's position, the identification of the number of cycles to reconstruct, and the prediction of the cycle model used to generate the missing cycles. We propose the reconstruction of the blood pressure signals recorded to cardiomyopathy patients and healthy subjects, using finger cuff.

\section{REAL DATA}

The reconstruction method proposed was applied to blood pressure signals from 10 cardiomyopathy patients and 10 healthy subjects. These records are part of two databases: 
HERIS - recorded at the Santa Creu i Sant Pau hospital, in Barcelona, Spain, according to a protocol previously approved by the Hospital Ethics Committee [10], and HEALTH - recorded at the Institute for Bioengineering of Catalonia [11]. All subjects gave their written consent to participate in the study.

The patients and healthy subjects were recorded during 30 min in supine position. The patients were registered at a sampling frequency of $1600 \mathrm{~Hz}$. On the other hand, the healthy subjects were registered at a sampling frequency of $500 \mathrm{~Hz}$. All signals were resampled at $250 \mathrm{~Hz}$. A wavelet based denoising algorithm was applied to all the signals [12], and their linear trend was removed. Figure 1 shows an example of these signals affected by artifacts due to calibration.
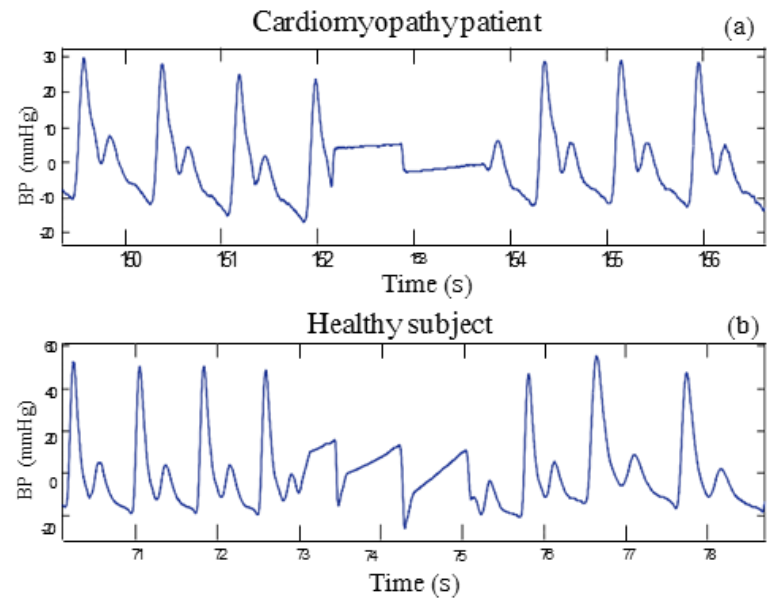

Figure 1. Blood pressure signal from (a) Cardiomyopathy patient, and (b) Healthy subject

\section{MethodOLOGY}

\section{A. Cycle detection}

After signal preprocessing, the position of each cycle is obtained using a wavelet decomposition method for marking each local minimum of the signal. The position of each event is represented by the vector

$$
E=\left[\begin{array}{lll}
E_{1} & \cdots & E_{N}
\end{array}\right],
$$

where $N$ is the number of events detected in the signal. This process includes physiological cycles and artifact episodes.

\section{B. Artifact detection}

To analyze the morphology of cycles and artifacts, the area under the curve of each one is calculated. In order to identify the artifacts, a parameter $\Theta_{E}$ is defined as the sum of the difference between these areas $\left(A_{i}\right)$, for each event analyzed and its following $M$ neighbours:

$$
\Theta_{E_{(i)}}=\sum_{j=i}^{i+M}\left|A_{i}-A_{j+1}\right|, \quad \forall i=1, \cdots, N,
$$

where $M$ is the number of neighbour events considered.

To select the optimum $M$, the parameter $\Theta_{E}$ is evaluated for different values of $M$. Figure 2 presents an example of (a) a real signal with some cycles and artifacts, and (b) the parameter $\Theta_{E}$ evaluated for different values of $M$. When the events are similar, this parameter presents lower value, whereas if the comparison is between a cycle and an artifact, its value is higher. According to this test, $M=10$ is selected. In this example, we can observe that with $M=2$ or 5 , values of $\Theta_{E}$ are similar comparing physiological cycles and cycles with artifacts. Therefore, the threshold of 10 is enough to differentiate between cycles and artifacts segments.

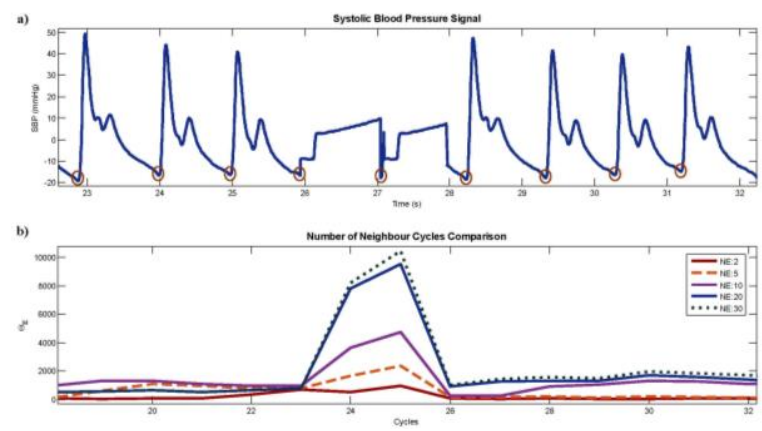

Figure 2. Excerpt of real blood pressure signal with (a) cycles and artifacts and (b) the diference beetween area under the curve of the events

This process is repeated for each cycle. The difference between a physiological cycle and an artifact is defined by an adaptive threshold $\left(T h_{\Theta_{E}}\right)$ as a function of the mean value and 3 standard deviation of $\Theta_{E}$ parameter:

$$
T h_{\Theta_{E}}=\overline{\Theta_{E}}+3 * S D\left(\Theta_{E}\right)
$$

To identify the first correct cycle in the signal, the 10 initial events are analyzed, comparing the difference between the areas of each event and their neighbours. This cycle is selected if the difference of these areas is less than or equal $T h_{\Theta_{E}}$. Finally, this cycle is the basis to determine the thresholds, to identify each artifact, and to generate the new reconstruction cycle.

The artifact events can have different lengths. To guarantee that all are detected, the process of identification is repeated $k$ times. In each iteration, the length of the maximum artifact detected is compared with the one in the iteration immediately before. The position of this artifact is fixed and excluded for the next iteration. Figure 3 presents an example of this process: (a) represents an excerpt of a real BP signal with different artifacts; (b) presents the first iteration, the amplitudes of $\Theta_{E}$ for each event; (c) and (d) present the next iterations, in each one discarding the artifacts overpass the threshold $T h_{\Theta_{E}}$; and so successively until there are no amplitudes overpassing the corresponding threshold. In this case, three iterations are enough to identify 
all artifacts to reconstruct, since there are no amplitudes overpassing its threshold $T h_{\Theta_{E}}$.

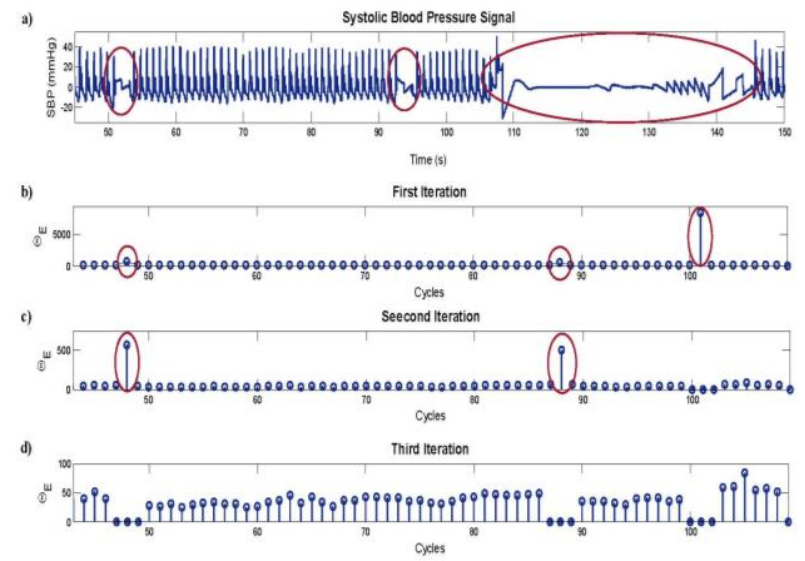

Figure 3. (a) Excerpt of a real BP signal with different episodes of artifacts, (b) the first iteration shows the amplitude of $\Theta_{E}$ for each even, (c) and (d) iterations present next amplitudes of $\Theta_{E}$ discarding the artifacts overpass the threshold $T h_{\Theta_{E}}$.

\section{Reconstruction}

The reconstruction of the artifact is based on the information of the neighbouring cycles of the original signal.

- The first step is to determine the length of the episode (le) to reconstruct, and how many cycles will be used. This number of cycles is calculated from the mean value of the neighbouring cycles and the length of the episode.

- Next step is to generate these cycles, using the same number of physiological cycles to reconstruct, of the left and the right of the original signal.

The segment is then replaced by crossfading the two extrapolated values, using the following window $[13,14]$

$$
w(n)= \begin{cases}1-\frac{1}{2}(2 u(n))^{\alpha}, & u(n) \leq \frac{1}{2} \\ \frac{1}{2}(2-2 u(n))^{\alpha}, & u(n)>\frac{1}{2}\end{cases}
$$

were $u(n)=(n+1) / l e, n=1, \ldots$, le. Crossfading is carried out by multiplying the forward extrapolated sequence by $w(n)$ and the backward extrapolated sequence by $1-w(n)$. A linear down-slope is attained with $\alpha=1$, whereas a step-like transition results when a $\alpha \rightarrow \infty$. The continuous slope of the window was obtained by $\alpha=3$.

Cycles with a value greater than $T h_{\Theta_{E}}$ are discarded for the reconstruction of the signal, and the nearest cycle below the threshold is used instead.

Figure 4 presents an example of the reconstruction process, when the cycle is compared with an artifact (a), and when the events analyzed are two physiological cycles (b).
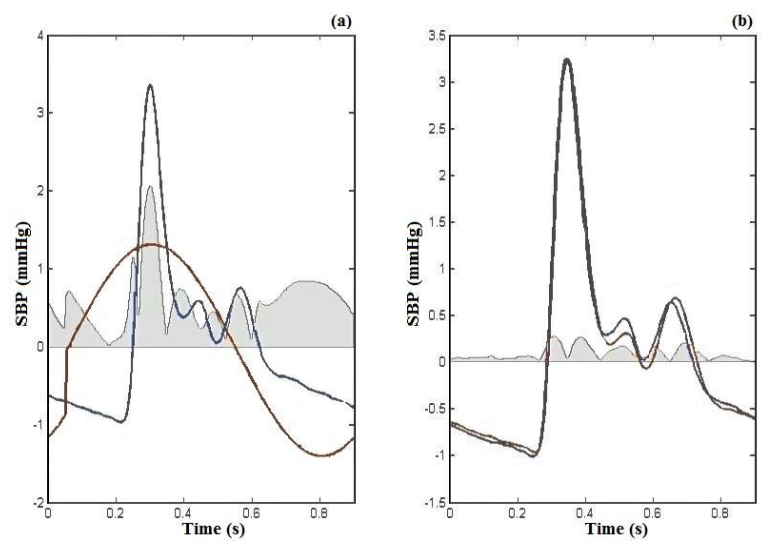

Figure 4. An example of the reconstruction episodes when comparing the difference between the areas under the curve of (a) a cycle with an artifact, and (b) two physiological cycles

\section{RESULTS}

To evaluate the performance of the proposed method, we simulated random episodes of artifacts into an excerpt of clean real signal. Then, the reconstruction process was applied. Figure 5 is an example of this simulation. We can observe the superposition of the reconstructed cycles over the originals. After visual inspection, we observe that the reconstructed cycles are similar to the original.

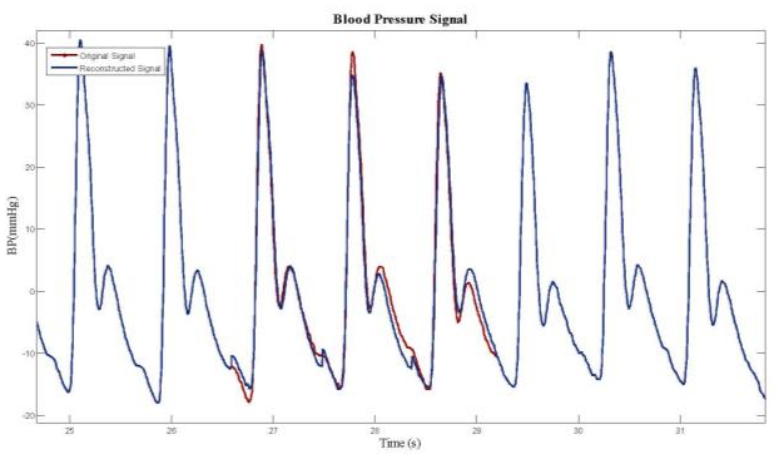

Figure 5. Superposition of the reconstructed cycles over the originals

The next step was to apply the reconstruction process to blood pressure signals of 10 cardiomyopathy patients and 10 healthy subjects. Figure 6 shows an example where an artifact was reconstructed by three physiological cycles. The length of the artifact episode is modeled with the neighbouring cycles of the left and right.

The quality of the reconstruction process was also evaluated comparing:

- the number of artifacts detected in function of the total artifacts $($ Detc),

- the number of cycles detected as artifacts in function of the total cycles $(\mathrm{C} w r)$,

- the number of artifacts no correctly reconstructed in function of the total artifacts $(R w r)$. 


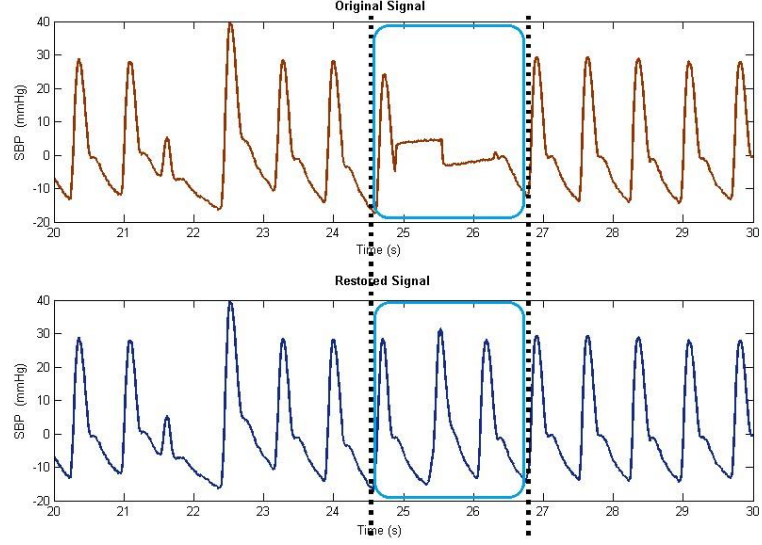

Figure 6. (a) Original blood pressure signal, and (b) the same signal with the reconstructed artifact episode

The results of this quality control were made through visual inspection. Table 1 shows a summary of these parameters for both datasets, represented by the mean values.

TABLE I. SUMARY OF THE RECOSNTRUCTION PROCESS

\begin{tabular}{cccc}
\hline Dataset & Detc (\%) & Cwr (\%) & Rwr (\%) \\
\hline Patients & 100 & 0.10 & 0.74 \\
Control & 100 & 0.65 & 0 \\
\hline
\end{tabular}

According to these results, all artifacts were correctly detected. On the other hand, cycles detected as artifacts and the artifacts incorrectly reconstructed were less than $1 \%$.

\section{CONCLUSION}

We have proposed a novel method to reconstruct artifacts episodes present in blood pressure signals. This method is based on obtain a cycle model from the neighbouring morphological cycles, maintaining the continuity of the signal. An adaptive threshold to identify these artifacts is defined.

The reconstruction quality was measured calculating the error between cycles and artifacts detected, in function of each total. This measurement was made visually. In all cases, these differences were less than $1 \%$.

This reconstruction process allows to maintain the dynamic of the signal, and consequently the dynamic of the system that represent. It is very helpful to verify that the artifact reconstruction is not distorting this dynamic, checking the cycles reconstructed have the expected amplitude and shape. For as, one proposes is to introduce a quantitative method for evaluate the quality of this process.

The calculations and simulations proposed allow to illustrate several types of problems that may be find recording quasi-periodic biomedical signals. Then, these processes can be included when these signals are studied.

Some of the interesting questions for further research include the generalization of the method for the reconstruction of other type of physiological signals. Summarizing, our propose contribute to minimizing the problems generated by the different interferences in the acquisition systems of the biomedical signals.

\section{REFERENCES}

[1] G. Ganeshapillai and J. Guttag, Template Matching for Physiological Signal Reconstruction., Massachusetts Institute of Technology, 2010.

[2] G. Ganeshapillai and J. Guttag, Real time reconstruction of quasiperiodic multiparameter physiological signals, Journal on Advances in Signal Processing, 2012, 2012:173.

[3] H. Zeng and A. Song, Removal of EOG Artifacts from EEG Recordings Using Stationary Subspace Analysis, The Scientific World Journal, 2014.

[4] C. Zhang, L. Tong, Y. Zeng, J. Jiang, H. Bu, B. Yan and J. Li, Automatic Artifact Removal from Electroencephalogram Data Based on A Priori Artifact Information, BioMed Research International,2015.

[5] N. Narendra and A. Guruva, Removal of ECG Artifact from EEG data using Independent Component Analysis and S-Transform, International Journal of Science, Engineering and Technology Research,2016, 5:712-716.

[6] R. Mahajan and B. Morshed Unsupervised Eye Blink Artifact Denoising of EEG Data with Modified Multiscale Sample Entropy, Kurtosis, and Wavelet-ICA, IEEE Journal of Biomedical and Health Informatics, 2014, 19,158-165.

[7] M. Mannan, M. Joeong and M. Kamran, ybrid ICA—Regression: Automatic Identification and Removal of Ocular Artifacts from Electroencephalographic Signals, Frontiers in human neuroscience,2016, 10:1- 17.

[8] M. Seyed, A. Salehizadeh,D. Dao, J. Bolkhovsky, C. Cho, Y. Mendelson and H. Chon, A Novel Time-Varying Spectral Filtering Algorithm for Reconstruction of Motion Artifact Corrupted Heart Rate Signals During Intense Physical Activities Using a Wearable Photoplethysmogram Sensor Sensors, Sensors, 2016,16:1-20.

[9] D. Tang, Y. Goh,M. Wong, and L. Lew, PPG signal reconstruction using a combination of discrete wavelet transform and empirical mode decomposition, 6th International Conference on Intelligent and Advanced Systems (ICIAS), 2016.

[10] A. Arcentales, P. Rivera, P. Caminal, A. Voss, A- Bayés-Genís, B.F. Giraldo, Analysis of Blood Pressure Signal in Patients with different Ventricular Ejection Fraction using Linear and Non-linear Methods, $38^{\text {th }}$ Annual International Conference of the IEEE Engineering in Medicine and Biology Society, 2016. pp. 2700-2703.

[11] Giraldo B.F., A. Calvo, B. Martínez, A. Arcentales, R. Jané, S. Benito, Blood Pressure Variability Analysis in Supine and Sitting Position of Healthy Subjects, XIII Mediterranean Conference on Medical and Biological Engineering and Computing MEDICON, 2013. pp 10211024.

[12] J.S. Sahambi, S.N. Tandonz5 R.K.P. Bhatt, Using Wavelet Transforms for ECG Characterization. IEEE Engineering in Medicine and Biology. 1997, pp 77-83.

[13] A. Garde, L. Sömmo, R. Jané and BF. Giraldo, Breathing pattern characterization in chronic heart failure patients using the respiratory flow signal, Annals of Biomedical Engineering, 2010: 38(12):3572-80

[14] P. Esquef, V. Valimaki,K. Roth, and I. Kauppinen, Interpolation of long gaps in audio signals using the warped Burg's method, 6th International Conference on Digital Audio Effects, 2003. pp. 1-6. 